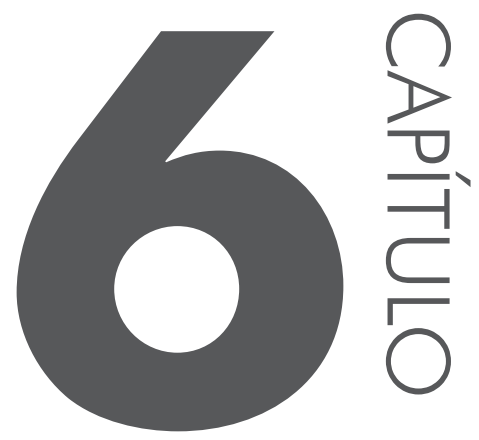

\title{
APLICATIVOS PARA DISPOSITIVOS MÓVEIS APLICADOS À SEGURANC̣A PÚBLICA
}

Lorielcio da Silva Peres

Roderval Marcelino

Vilson Gruber

João Bosco da Mota Alves

\section{INTRODUC̣ÃO}

A informação é um dos recursos mais importantes para a tomada de decisão. A grande questão é: como adquirir informação? A tecnologia é atualmente utilizada para os mais diversos objetivos, entre eles a busca e organização da informação. Somente ter informação não basta; é necessário que ela seja divulgada, comunicada às partes interessadas, para que seja útil em tomadas de decisão, 
que nos dias de hoje requerem rapidez e eficiência. Para isso, faz-se necessário ter sempre disponíveis mecanismos de obtenção, processamento e divulgação de informações ABDI (2010).

As Tecnologias de Informação e Comunicação (TIC) reúnem todo esse arcabouço de requisitos para prover a comunidade com informações para as tomadas de decisões necessárias ao crescimento e ao desenvolvimento do que chamamos hoje de Sociedade da Informação. "A introdução das TIC em todas as áreas de atividades, de geradora de externalidades positivas, passou nos anos recentes a constituir o cerne do que se vem chamando de Sociedade da Informação" (ABDI, 2010, p. 13).

Foi com o objetivo de gerar e organizar informação que aconteceu, em 2010, a Oficina de Trabalho "Visão de Futuro e Agenda TIC: 2008-2025", iniciativa da Agência Brasileira de Desenvolvimento Industrial (ABDI) em conjunto com o Centro de Gestão e Estudos Estratégicos (CGEE). O trabalho foi desenvolvido por um grupo de especialistas em aplicações mobilizadoras.

Aplicações mobilizadoras referem-se à oferta de produtos e serviços de informação e comunicação que considerem estratégias globais no acesso e uso de tecnologias de informação e comunicação (TICs) e que demandem desenvolvimentos de novas tecnologias, equipamentos, metodologias, modelos de negócio, em um esforço articulado por parte das instituições acadêmicas, de governo e empresas (ABDI, 2010, p. 12).

Este trabalho tem o objetivo contribuir com os estudos relativos à aplicações mobilizadoras desenvolvidas a partir das TIC aplicadas à segurança pública nacional, visando gerar informações que poderão ser utilizadas em aplicações e que demandem mais estudos, aperfeiçoamento, investimentos e estratégias de uso e gerenciamento.

Existe um conjunto de processos que podem ser melhorados a partir de novas aplicações e tecnologias como:

- Atendimento e interação com o cidadão, envolvendo todos os canais de comunicação e troca de informações diretamente com a sociedade, seja para registro de ocorrências, difusão de informações ou prestação de serviços ao cidadão.

- Sistemas de tratamento de ocorrências, envolvendo todos os trâmites desde os registros do incidente até a finalização de processos judiciais.

- Tratamento de informações relativas à identificação civil e criminal, incluídas aí todas as formas de coleta, armazenamento e processamento de dados de identificação.

- Tratamento de informações coletadas por serviços de inteligência e integração dos sistemas de informação das diversas organizações policiais. 
- Acompanhamento e avaliação dos procedimentos realizados pelos órgãos de segurança. (ABDI, 2010 p. 56)

Foi baseando neste conjunto de processos que este trabalho se orientou para buscar mais informações relacionadas às aplicações tecnológicas para a segurança pública.

No desenvolvimento de software, atualmente está acontecendo uma revolução nos termos do que entendemos por computação móvel, os smartphones como o iPhone e outros que utilizam o sistema operacional Android redefiniram o que entendemos por computação móvel.

Uma aplicação móvel pode ter milhões de descarregamentos em pouco tempo e esse mercado potencial gerou uma nova forma de distribuição e entrega de software a partir das lojas virtuais de apps (aplicativos), que reduzem significativamente os custos de distribuição e de comercialização. "Os mercados para aplicativos móveis criaram um mecanismo totalmente novo para a distribuição de conteúdo, estimulando investimentos consideráveis no desenvolvimento de software para dispositivos móveis" (UNESCO, 2014, p. 23).

Diante da realidade atual, em relação às aplicações móveis, optou-se por limitar a investigação a tecnologias disponíveis para dispositivos móveis do tipo smartphones e tablets.

\section{DESENVOLVIMENTO}

As aplicações mobilizadoras referem-se à oferta de produtos e serviços de informação e comunicação, relacionando com estratégias para acesso e uso que demandem desenvolvimentos de novas tecnologias, equipamentos e metodologias. A ideia para este trabalho se consolidou com a perspectiva de pesquisar uma tecnologia bastante atual, que é a utilização de dispositivos móveis como smartphones e tablets a partir de aplicativos (softwares) desenvolvidos para atender a objetivos específicos. Os objetivos norteadores deste trabalho foram identificar o cenário nacional deste conjunto de tecnologia, equipamentos e metodologia e sua capacidade em gerar informação que podem ser úteis à segurança pública e obter mais conhecimentos quanto aos aplicativos e aos dispositivos móveis e suas destinações junto à sociedade.

Segundo a ABDI (2010), existe um conjunto de processos que podem ser melhorados a partir de novas aplicações e tecnologias, e está relacionado ao atendimento e à interação com o cidadão, envolvendo todos os canais de comunicação e troca de informações diretamente com a sociedade, seja para registro de ocorrências, difusão de informações ou prestação de serviços. Paralelo à questão 
das aplicações mobilizadoras, a pesquisa exploratória apontou para um novo cenário em termos de desenvolvimento de software, no qual, atualmente, está acontecendo uma revolução em termos do que se pode entender por computação móvel. Por meio de tablets e smartphones, uma aplicação móvel pode ter milhões de descarregamentos em pouco tempo e esse mercado potencial gerou uma nova forma de distribuição e entrega de software por meio das lojas virtuais de apps. Esse formato de distribuição reduz significativamente os custos de distribuição e comercialização, o que aponta para uma nova metodologia tanto no sentido de busca de ferramentas, quanto na própria utilização. O mercado também abre um novo leque de tecnologia a ser investido pelos órgãos públicos e pelo governo federal por meio de pesquisa e de desenvolvimento.

A ABDI e o CGEE selecionaram como ponto de partida para a construção da visão de futuro sete aplicações, considerando os objetivos dos cinco subprogramas do Programa Mobilizador em TIC, da Política de Desenvolvimento Produtivo, e pelo menos três destes subprogramas que estão relacionados ao que foi pesquisado neste trabalho, que são: software e serviços de TI, infraestrutura para inclusão digital e adensamento da cadeia produtiva. Entre as sete aplicações encontram-se os sistemas aplicados à segurança pública, ou seja, no cenário nacional de aplicativos móveis foi possível identificar aplicativos que correspondem aos objetivos dos subprogramas.

A rotina de trabalho relacionada à segurança pública é bastante dinâmica e precisa de agilidade. Uma tecnologia que disponha de mobilidade com certeza trará facilidades e praticidade para o dia a dia. A mobilidade ou computação móvel refere-se basicamente à capacidade de um dispositivo poder ser movimentado fisicamente enquanto ele está em uso. Aplicativos móveis são softwares específicos para dispositivos móveis como tablets e smartphones; alguns aplicativos vêm instalados nos dispositivos e outros podem ser adquiridos nas lojas de aplicativos na internet como, App Store, Google Play ou Windows Phone Store. Os aplicativos pesquisados e ofertados pelo governo estão disponíveis nestas lojas e, em sua maioria, disponíveis para pelos menos dois sistemas operacionais: Android e IOS.

A pesquisa procurou se embasar em aplicativos ofertados pelo Governo Federal para traçar um cenário nacional. O governo criou um guia de aplicativos, um catálogo de aplicativos para dispositivos móveis desenvolvidos por órgãos do Poder Executivo federal. Esse guia é um portal centralizador no qual o cidadão pode obter informações e conhecer os aplicativos que o governo oferece. Todos os aplicativos disponibilizados são gratuitos e de responsabilidade de órgãos de governo. Para acessar a relação, basta acessar o link: <http://www.aplicativos. gov.br/>, no qual é possível fazer uma busca por órgãos, conhecer e acessar links para download. Este portal é fácil de localizar a partir de uma busca no Google ou outro sistema de busca, e a forma de apresentação dos aplicativos também é 
simples e objetiva. No portal, os aplicativos podem ser agrupados a partir dos destaques, por categoria, por órgão ou pela plataforma.

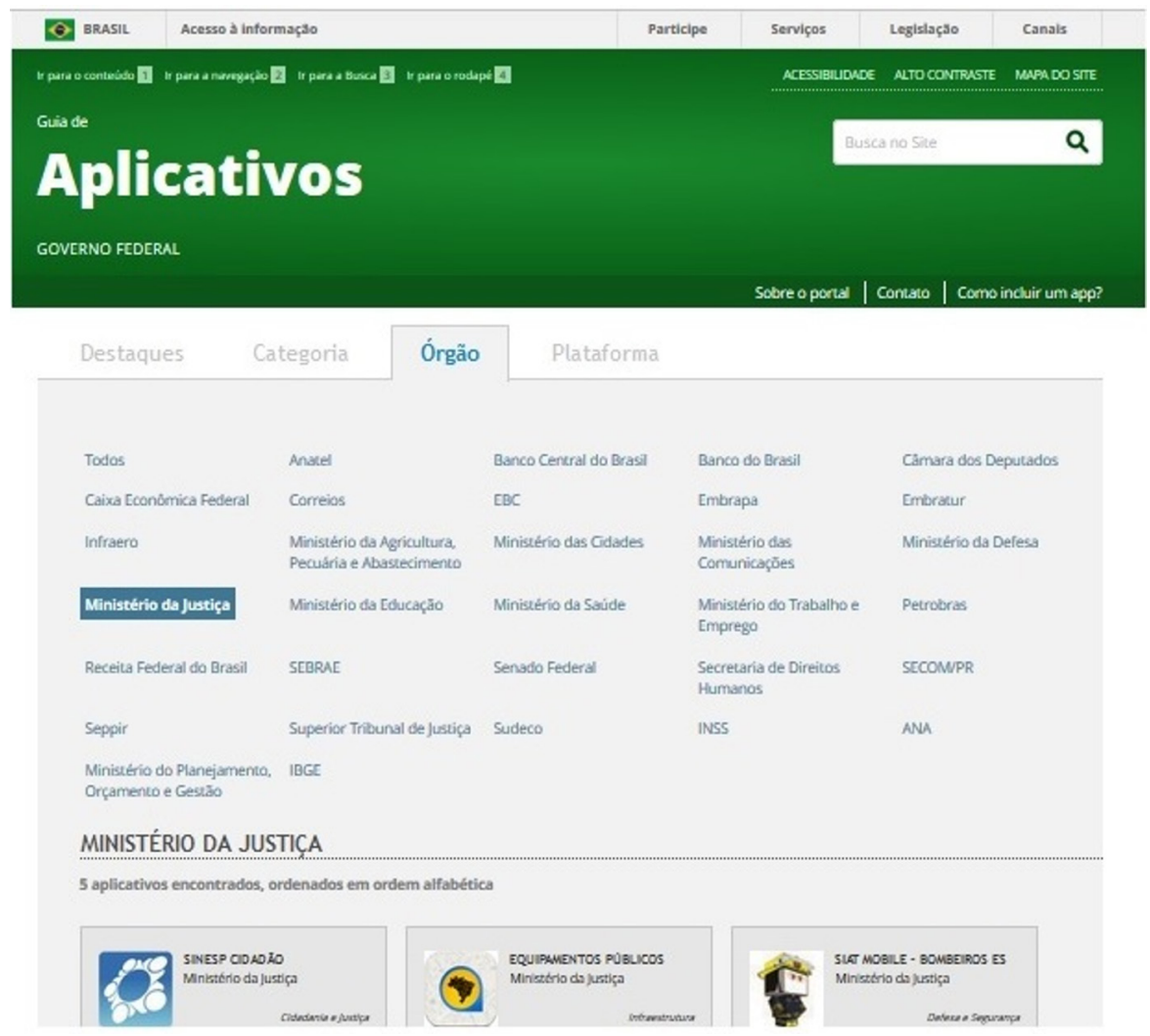

Figura 6.1 Guia de aplicativos do Governo Federal - agrupamento por órgão.

Fonte: http://www.aplicativos.gov.br/orgaos?filtro=Orgao\&valor=123f54b2 1bf066a578a889e932d9ef1 1 .

As considerações a seguir, relacionadas aos aplicativos, buscam, além de pontuar questões observadas nesses aplicativos, apresentar alguns aplicativos similares ofertados ao mercado por outros órgãos públicos e até por algumas iniciativas privadas. Complementando o cenário nacional ofertado pelo governo, o estudo visa orientar escopos de novas pesquisas, mais direcionadas à análise de conteúdo, e recursos para procurar aperfeiçoar os aplicativos já existentes e direcionar a pesquisa e desenvolvimento de novos aplicativos.

O aplicativo do Ministério da Justiça (Sinesp Cidadão), por exemplo, tem uma série de outros aplicativos que se assemelham. Foi possível identificar, por meio da pesquisa, que uma das funções do aplicativo do Governo Federal é auxiliar o cidadão na consulta a veículos a partir da placa e tem vários similares desenvolvidos pelos Departamentos Estaduais de Trânsito (Detran), como: 


\section{Aplicativo Detran.SP}

Aplicativo utilizado para consultar informações sobre seu próprio veículo ou de terceiros, e para consulta de informações de Carteira Nacional de Habilitação $(\mathrm{CNH})$. É oferecido pela Companhia de Processamento de Dados do Estado de São Paulo (Prodesp). Disponível em: <https://play.google.com/store/apps/ details?id=br.gov.sp.detran.consultas $>$.

Com o novo aplicativo do Detran.SP para tablets e smartphones, ficou ainda mais fácil verificar a pontuação de sua $\mathrm{CNH}$ e possíveis multas registradas em seu veículo. Para ter acesso às consultas, basta utilizar a mesma senha do portal do Detran.SP.

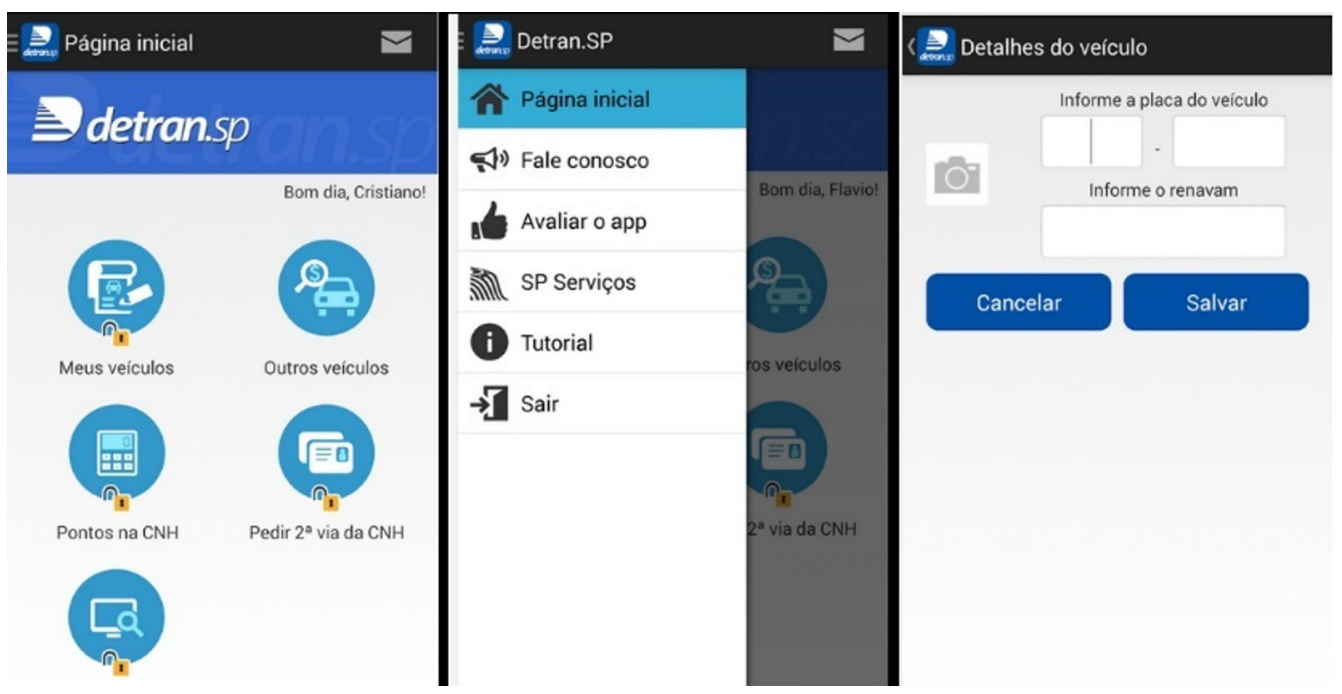

Figura 6.2 Aplicativo Detran.SP.

Fonte: https://play.google.com/store/apps/details?id=br.gov.sp.detran.consultas.

\section{Aplicativo Detran CE Mobile}

Aplicativo utilizado para consultar a situação de veículos a partir da placa e/ ou RENAVAM, entre outros. É oferecido por Detran Ceará. Disponível em: <https://play.google.com/store/apps/details?id=br.gov.ce.detran.mobile>.

Este é o mais novo aplicativo do DETRAN Ceará. Com ele, os usuários de smartphones e tablets obtém acesso rápido e amigável a:

- Informações da Carteira Nacional de Habilitação (pontuação, vencimento e renovação).

- Consulta de veículos pela placa e/ou Renavam. 
- Geração de código de barras para pagamento de taxas via internet Banking.

- Informações sobre transporte rodoviário intermunicipal de passageiros (ônibus e vans)

- Envio de críticas, reclamações e denúncias diretamente para a ouvidoria do órgão [...].
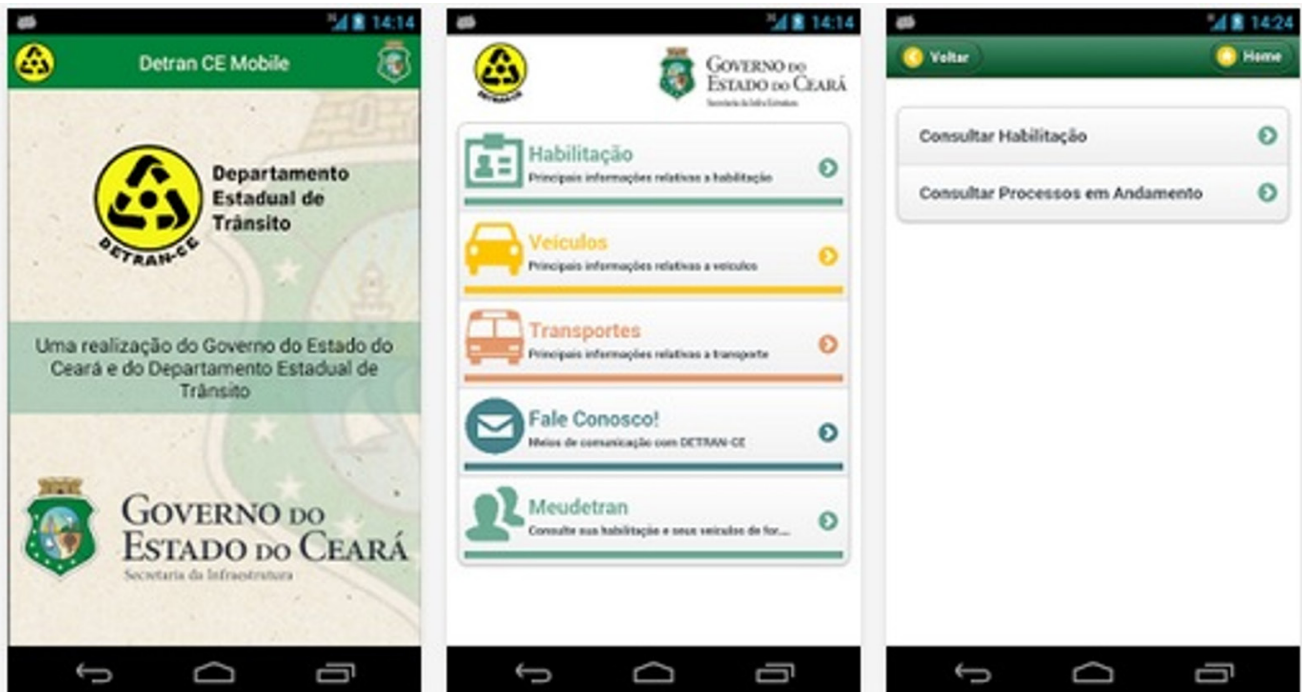

Figura 6.3 Aplicativo Detran CE.

Fonte: https://play.google.com/store/apps/details?id=br.gov.ce.detran.mobile.

Analisando os aplicativos dos órgãos estaduais e comparando com o aplicativo do governo, foi possível levantar algumas considerações importantes, iniciando pela questão de existirem vários aplicativos diferentes, mas basicamente com a mesma destinação de uso para cada estado. A primeira consideração a ser fazer é em relação aos custos de pesquisa e desenvolvimento despendidos por todos os órgãos estaduais. Nesta mesma linha de raciocínio, segue a questão da informação, que é descentralizada e limitada por estado, ou seja, cada aplicativo estadual consulta bases de dados diferentes dos veículos do seu estado, como Detran e Prodesp, enquanto o aplicativo do governo consulta uma base nacional do Departamento Nacional de Trânsito (Denatran).

Outra observação que cabe é em relação às funcionalidades acessórias, ou seja, o que um aplicativo do governo oferece em relação ao que cada aplicativo estadual oferece. No aplicativo do governo (Sinesp Cidadão), é possível, além da consulta de veículos, consultar desaparecidos e mandados de prisão. Nos aplicativos do Detran dos estados, além da consulta de veículos, comum a todos, outras destinações são apresentadas, como consultar informações sobre seu próprio veículo, sobre $\mathrm{CNH}$ e pontuação, solicitar segunda via de $\mathrm{CNH}$, informações sobre transporte, geração de código de barras para pagamento de taxas, entre outros. 
É importante ponderar a respeito de como o resultado da consulta é apresentada: alguns aplicativos apresentam os dados de forma bem sintética, enquanto outros o fazem com um nível de detalhamento mais aprofundado, o que oferece mais subsídios e informações tanto para o trabalho dos agentes de segurança pública como para a utilização do cidadão civil.

Com isso, é possível afirmar que não existe um padrão de informação, e que se houvesse uma interação entre o governo e os estados seria possível otimizar tanto a questão de investimentos em pesquisa e desenvolvimento, quanto outras questões relacionadas às fontes de informações, formas de apresentação e destinações de uso dos aplicativos.

Outro aplicativo do governo ao qual também cabe a mesma linha de considerações é o STJ - Consulta Processual, que é um aplicativo oficial do Superior Tribunal de Justiça (STJ) e permite acesso à consulta processual. Com funcionalidades e destinação de uso muito semelhantes a este aplicativo, existem vários outros aplicativos gratuitos ofertados por empresas privadas, destinados à consulta processual para vários estados. Em relação aos órgãos públicos, o Tribunal de Justiça do Estado do Pará lançou, por meio da Secretaria de Informática, o TJPA - Mobile .

\section{Aplicativo TJPA - Móbile}

Aplicativo utilizado para consulta processual e acompanhamento de processos. É oferecido por Tribunal de Justiça do Estado do Pará. Disponível em: $<$ https://play.google.com/store/apps/details?id=br.jus.tjpa.androidlawsuit>.

Realizar a Justiça buscando a excelência jurisdicional, contribuindo com o efetivo fortalecimento do Estado democrático de Direito.

- Consulta processual;

- consulta por número do processo;

- leitura por código de barras;

- consulta por nome da parte;

- consulta por número da $\mathrm{OAB}$;

- informações gerais, partes envolvidas, movimentações, custas, audiências, documentos $[\ldots]$.

Como é possível observar, a destinação e as funcionalidades são muito semelhantes ao aplicativo do Governo Federal. Neste caso, o que se pode ponderar é a respeito do padrão na apresentação das informações, dos recursos que podem ser incorporados a um ou a outro e, ainda, da base de pesquisa e desenvolvimento 
que pode servir como base para outros órgãos públicos investirem na tecnologia e metodologia de aplicações móveis.

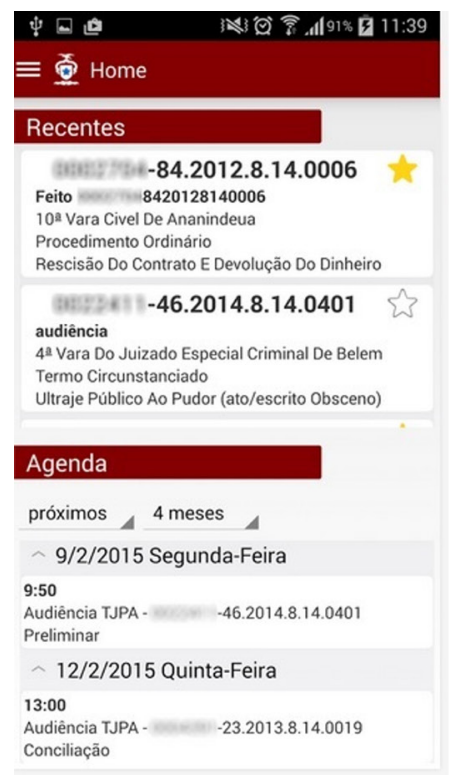
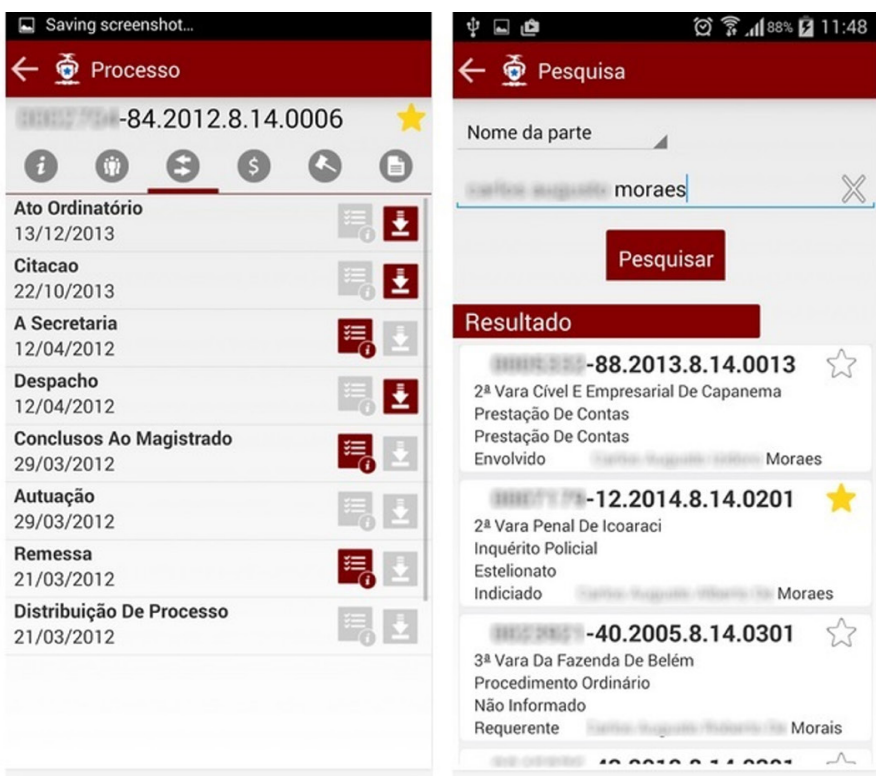

Figura 6.4 Aplicativo TJPA - Mobile.

Fonte: https://play.google.com/store/apps/details?id=br.jus.tipa. androidlawsuit.

\section{CONSIDERAC̣ÕES FINAIS}

Muitas considerações importantes podem ser apontadas em relação à pesquisa. Uma delas é o quanto às pequenas empresas e iniciativas de profissionais independentes aparecem com várias diversificações de aplicativos, com destinações muito semelhantes aos pesquisados, sendo ofertados, na maioria das vezes, gratuitamente nas lojas de aplicativos. Uma pesquisa que poderia ser feita é em relação às possíveis parcerias destes com os órgãos públicos para o desenvolvimento de aplicativos oficiais.

Outra característica importante nos aplicativos apresentados nesta pesquisa está relacionada à plataforma em que o app foi desenvolvido. A maioria dos aplicativos está disponível para os sistemas operacionais Android, utilizado por várias marcas de smartphones e tablets, baseado no núcleo Linux e atualmente desenvolvido pela Google; e IOS, da Apple. Também foi possível observar que, em relação aos sistemas operacionais, ainda não existe um padrão definitivo estabelecido, mesmo que maioria deles sendo desenvolvida para IOS e Android, alguns funcionam melhor em uma plataforma do que outra e as atualizações algumas vezes passam a ser geradas em apenas uma das plataformas. 
Como considerações finais, pode-se afirmar, pelo que foi encontrado nas pesquisas, que os dispositivos móveis e seus aplicativos vieram para ficar e estão compondo um novo cenário em termos de computação móvel, que a segurança pública já está inserida neste contexto, ainda que de forma embrionária, e que existe um campo bastante amplo para outras pesquisas, que possibilitam aprofundar o desenvolvimento de aplicações mobilizados das TIC para os sistemas de segurança pública.

\section{REFERÊNCIAS}

AGÊNCIA BRASILEIRA DE DESENVOLVIMENTO INDUSTRIAL - ABDI. Cadernos Temáticos - Tecnologias de Informação e Comunicação - TIC: Sistemas Aplicados a Segurança Pública. Brasília: Abdi, 2010.

CASTELLS, M. A sociedade em rede. São Paulo: Paz e Terra, 1999.

. A galáxia da internet: reflexões sobre a internet, os negócios e a sociedade. (Tradução de Maria Luiza X. A. Borges; revisão técnica de Paulo Vaz). Rio de Janeiro: Jorge Zahar, 2003.

EVANS, D. A internet das coisas: como a próxima evolução da internet está mudando tudo. 2011. Disponível em: <http://www.cisco.com/web/BR/assets/executives/pdf/ internet_of_things_iot_ibsg_0411final.pdf>. Acesso em: 29 maio 2015.

FURTADO, V. Tecnologia e gestão da informação na segurança pública. Rio de Janeiro: Garamond, 2003.

RICHARDSON, R. J. Pesquisa social: métodos e técnicas. 3.ed. São Paulo: Atlas, 1999. TORRES, C. E. Mobilidade: computação móvel, dispositivos e aplicativos. 2013.

Disponível em: <http://pt.slideshare.net/cetorres/palestra-mobilidade-computao-mveldispositivos-e-aplicativos-2013>. Acesso em: 14 maio 2015.

UNESCO. O futuro da aprendizagem móvel: implicações para planejadores e gestores de políticas. Brasília: Unesco, 2014. 64 p. ISBN: 978-85-7652-188-4. 